\title{
Characterization of mitosis-specific phosphorylation of tumor-associated microtubule-associated protein
}

\author{
Kyung Uk Hong, Hyun-Jun Kim, \\ Chang-Dae Bae ${ }^{1}$ and Joobae Park ${ }^{1}$ \\ Department of Molecular Cell Biology and \\ Samsung Biomedical Research Institute \\ Sungkyunkwan University School of Medicine \\ Suwon 440-769, Korea \\ ${ }^{1}$ Corresponding authors: Tel, 82-31-299-6130; \\ Fax, 82-31-299-6149; E-mail, jbpark@ med.skku.ac.kr (J.B. Park) \\ Tel, 82-31-299-6133; Fax, 82-31-299-6149; \\ E-mail, cdbae@med.skku.ac.kr (C.D. Bae) \\ DOI 10.3858/emm.2009.41.11.089
}

Accepted 8 July 2009

Abbreviations: Cdk1, cyclin-dependent kinase 1; CKAP2, cytoskeleton associated protein 2; GFP, green fluorescent protein; TMAP, tumor-associated microtubule-associated protein; WT, wild type

\begin{abstract}
Tumor-associated microtubule-associated protein (TMAP), also known as cytoskeleton associated protein 2 (CKAP2), has been recently shown to be involved in the assembly and maintenance of mitotic spindle and also plays an essential role in maintaining the fidelity of chromosome segregation during mitosis. We have previously reported that TMAP is phosphorylated at multiple residues specifically during mitosis, and characterized the mechanism and functional importance of phosphorylation at one of the mitosis-specific phosphorylation residues (i.e., Thr-622). However, the phosphorylation events at the remaining mitotic phosphorylation sites of TMAP have not been fully characterized in detail. Here, we report on generation and characterization of phosphorylated Thr-578- and phosphorylated Thr-596-specific antibodies. Using the antibodies, we show that phosphorylation of TMAP at Thr-578 and Thr-596 indeed occurs specifically during mitosis. Immunofluorescent staining using the antibodies shows that these residues become phosphorylated starting at prophase and then become rapidly dephosphorylated soon after initiation of anaphase. Subtle differences in the kinetics of phosphorylation between Thr-578 and Thr-596 imply that they may be under different mechanisms of phosphorylation during mitosis. Unlike the phosphoryla-
\end{abstract}

tion-deficient mutant form for Thr-622, the mutant in which both Thr- 578 and Thr- 596 had been mutated to alanines did not induce significant delay in progression of mitosis. These results show that the majority of mitosis-specific phosphorylation of TMAP is limited to pre-anaphase stages and suggest that the multiple phosphorylation may not act in concert but serve diverse functions.

Keywords: antibodies, polyclonal; cell cycle; CKAP2 protein, human; mitosis; phosphorylation

\section{Introduction}

Tumor-associated microtubule-associated protein (TMAP), also known as cytoskeleton associated protein 2 (CKAP2), has been previously identified as one of the gene products which are significantly elevated in various human malignancies (Maouche-Chretien et al., 1998; Eichmuller et al., 2001; Bae et al, 2003). However, TMAP lacks any known functional domains or motifs, and its cellular functions and relationship to tumorigenesis have remained elusive until recently.

Previously, we have demonstrated that TMAP plays important roles in mitosis. Its mRNA and protein levels begin to incline as cells enter the cell cycle and peaks at $\mathrm{G}_{2} / \mathrm{M}$ phases of the cell cycle (Whitfield et al., 2002; Jeon et al., 2006). TMAP has microtubule-stabilizing properties and during mitosis, it mainly localizes to the mitotic spindle apparatus (Jin et al., 2004; Hong et al., 2007). Because of these properties, it has been proposed that TMAP is one of the regulators of the spindle microtubule dynamics. In support of this notion, its overexpression results in bundling and elongation of spindle microtubules as well as defects in centrosome separation, which ultimately induces formation of monopolar spindles and subsequent arrest in mitosis (Hong et al., 2007). Also, TMAP is targeted for destruction by the anaphase promoting complex-Cdh1 as cells exit from mitosis, and its timely degradation is functionally important for proper establishment of bipolar spindles in the following cell cycle (Hong et al., 2007). In addition, we have recently shown that TMAP is required for keeping the fidelity of chromosome segregation and thus for maintaining genomic stability (Hong et al., 2009a). Depletion of TMAP in mammalian cells 
results in chromosome missegregation characterized by formation of chromatin bridges and deformed interphase nuclei. Defects in chromosome segregation induced by depletion of TMAP ultimately lead to reduced cell viability and development of aneuploidy. These indicate that TMAP serves important functions during mitosis.

It appears that the mitotic functions of TMAP are regulated by multiple phosphorylation events. Similar to other mitotic regulators, TMAP is hyper-phos-
A

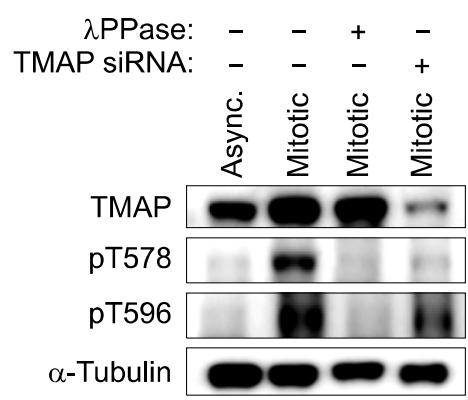

B

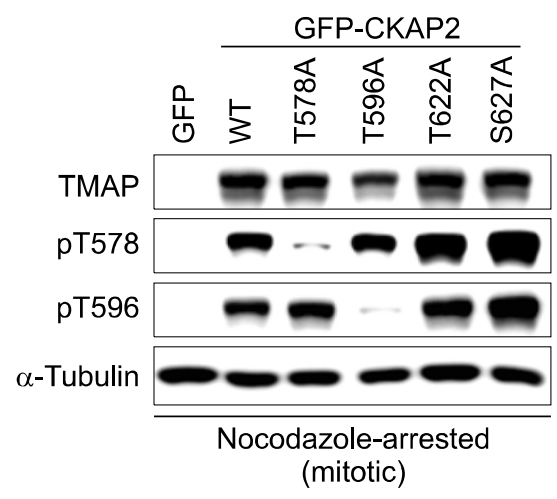

C

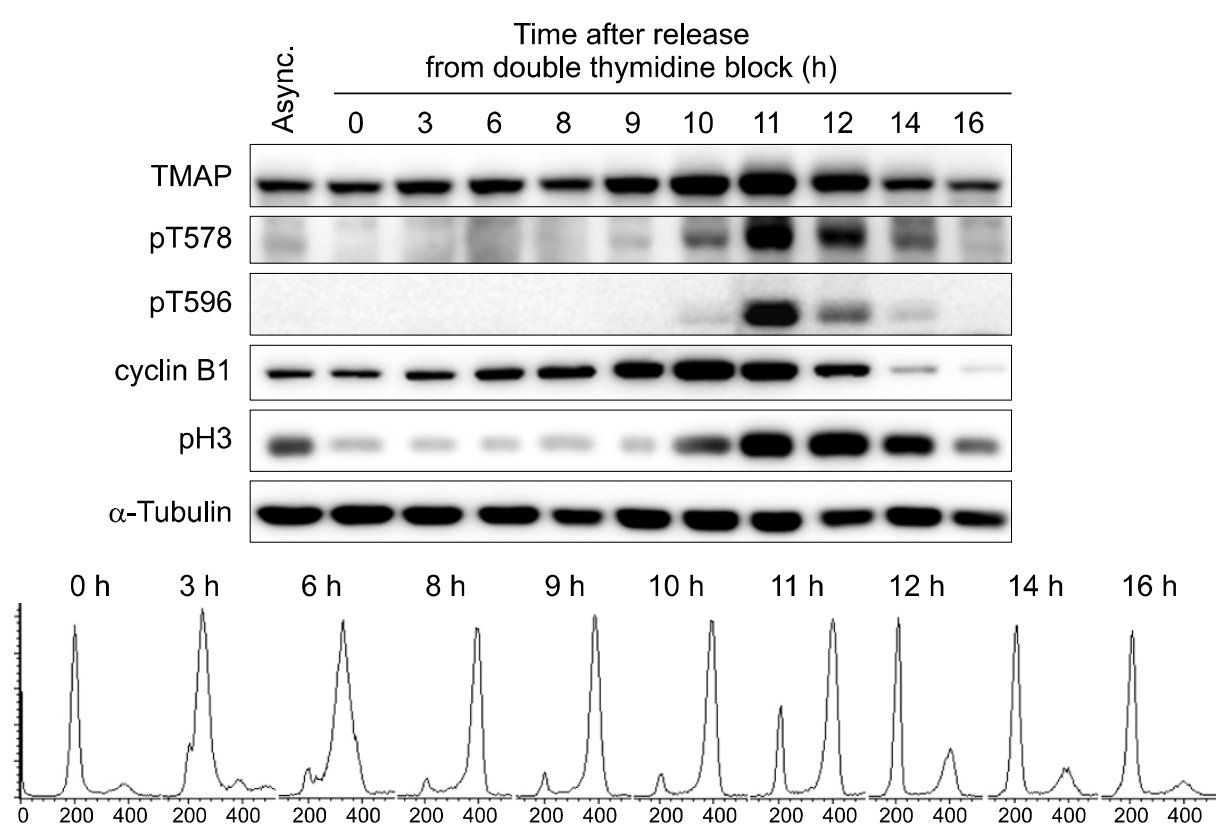

Figure 1. Generation and characterization of phosphorylated Thr-578- (pT578) and phosphorylated Thr 596 (pT596)-specific antibodies. (A) pT578 and pT596 antibodies detect endogenous TMAP in a phosphorylation-specific manner. Asynchronous (Async.) or nocodazole-arrested (Mitotic) HeLa cell ly-

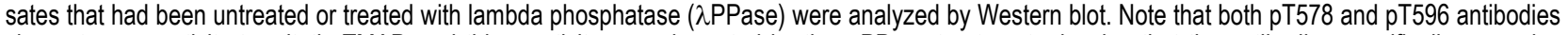
show strong reactivity to mitotic TMAP, and this reactivity was abrogated by the $\lambda$ PPase treatment, showing that the antibodies specifically recognize phosphorylated TMAP. TMAP siRNA-treated mitotic HeLa cell lysate was used to confirm the identity of the protein band detected by the antibodies. (B) HEK 293T cells were introduced with GFP or GFP-fused WT or indicated phosphorylation-deficient mutant, arrested in mitosis by nocodazole treatment, and analyzed by Western blot. Rabbit polyclonal antibody against human TMAP was used to show the levels of exogenously introduced GFP-fusion proteins. The reactivity of pT578 or pT596 antibody was abrogated specifically by the site-specific mutation, confirming the specificity of each antibody. $\alpha$-Tubulin served as a loading control. (C) Mitosis-specific phosphorylation of Thr-578 and Thr-596. HeLa cells synchronized by double thymidine block were released and harvested at indicated time points. At each time point, samples were analyzed by Western blot and flow cytometry to measure changes in the relative levels of pT578 and pT596 during the cell cycle. Cyclin B1 and phospho-histone H3 (pH3) were used as $\mathrm{G}_{2} / \mathrm{M}$ and mitosis markers, respectively. The windows of Thr-578 and Thr-596 phosphorylation coincide with that of pH3, indicating that phosphorylation at these residues occurs specifically during mitosis. 
phorylated during mitosis, causing a protein band shift on SDS-PAGE gel (Hong et al., 2008; 2009b). Previously, we have reported that at least 4 different residues located near the C-terminus (i.e., Thr-578, Thr-596, Thr-622, and Ser-627) are differentially phosphorylated during mitosis (Hong et al., 2009b). Among these, Thr-622 is phosphorylated specifically by Cdk1/cyclin B1 both in vitro and in vivo. Cdk1/cyclin B1-mediated phosphorylation of TMAP at Thr-622 contributes to proper establishment of functional bipolar spindles, presumably by influencing the rate of TMAP protein turnover at spindle microtubules.

The findings described above clearly indicate that TMAP plays important roles in mitosis and that the mitotic functions of TMAP are regulated by phosphorylation. However, the mechanisms and functional importance of phosphorylation of the remaining sites are completely unknown. In the present study, in order to study the kinetics of phosphorylation of Thr-578 and Thr-596 in detail and to provide tools for functional studies on mitotic phosphorylation of TMAP, we generated and characterized phospho-Thr-578- and phospho-Thr596-specific antibodies. Using these antibodies, we examined the kinetics of phosphorylation of each residue during the cell cycle and the subcellular localization of phosphorylated protein during mitosis. Also we report our findings on the effects of different phosphorylation-deficient mutants on progression of mitosis.

\section{Results}

\section{Generation and characterization of phosphorylated Thr-578- and phosphorylated Thr-596-specific antibodies}

In order to characterize the phosphorylation events occurring at Thr-578 and Thr-Thr-596 of TMAP in more detail, we regenerated affinity-purified rabbit polyclonal antibodies that are specific for each of the phosphorylated residue using synthetic peptides as described in Methods. We first tested the specificity of the antibodies by Western blot. Both phosphorylated Thr-578 (pT578) and phosphorylated Thr-596 (pT596) antibodies reacted specifically with the TMAP protein band from the mitotic cell lysate, and the immunoreactivity was either abolished or markedly reduced by lambda phosphatase treatment of the mitotic cell lysate or upon siRNA-mediated depletion of TMAP (Figure 1A). This indicates that both antibodies specifically recognize phosphorylated forms of TMAP in mitotic cells. Next, we tested if each antibody reacted with the phosphorylated form of TMAP in a site-specific manner by testing its reactivity towards wild type (WT) or a panel of mutant forms of TMAP in which one of the four mitotic phosphorylation sites had been mutated to alanine. For pT578 antibody, mutation of Thr-578 to alanine caused loss of its reactivity to GFP-fused TMAP protein, while mutations at Thr-622, Thr-596, and Ser-627 did not affect its reactivity (Figure $1 \mathrm{~B}$ ). Similarly, the reactivity of pT596 antibody to GFP-TMAP was lost by mutation at Thr-596 alone (Figure 1B). Taken together, these data demonstrate that each antibody is specific for the epitope containing the designated phosphorylated residue located within TMAP protein.

\section{Cell cycle-dependent changes in the level of Thr-578 and Thr-596 phosphorylation}

Next, we used the pT578- and pT596-specific antibodies to measure the changes in the relative levels of Thr-578 and Thr-596 phosphorylation during the cell cycle. HeLa cells synchronized by double thymidine block were released and harvested at regular time intervals for Western blot and flow cytometric analyses. As previously reported (Jeon et al., 2006), TMAP protein level gradually inclined as cells progressed through $S$ phase and enter $G_{2}$ and $M$ phases of the cell cycle, and the pattern of its expression was similar to that of cyclin B1, a $\mathrm{G}_{2} / \mathrm{M}$ cyclin (Figure $1 \mathrm{C}$ ). In contrast, pT578 and pT596 levels remained undetectable up to $G_{2}$ phase but dramatically increased as cells entered mitosis. As cells exited mitosis, the phosphorylation levels declined rapidly and became undetectable (Figure 1C). Although the patterns of Thr-578 and Thr-596 phosphorylation during the cell cycle were quite similar to that of histone $\mathrm{H} 3$ phosphorylation which is a well-known marker of mitosis (Hendzel et al., 1997), the window of Thr-578 and Thr-596 phosphorylation was relatively narrower than that of histone $\mathrm{H} 3$ phosphorylation (Figure 1C). Of note, de-phosphorylation of Thr-578 and Thr-596 occurred concomitantly with cyclin B1 degradation. These results indicate that Thr-578 and Thr-596 phosphorylation occurs specifically during mitosis and suggest that the window of Thr-578 and Thr-596 phosphorylation may be restricted to relatively earlier phases of mitosis.

\section{Subcellular localization of phosphorylated TMAP during mitosis}

In order to monitor the timing of Thr-578 and Thr-596 phosphorylation in detail and to characterize the subcellular localization pattern of phos- 
phorylated TMAP during mitosis, we performed immunofluorescence staining of HEK 293 cells using pT578- and pT596-specific antibodies. Both antibodies resulted in strong staining specifically in mitotic cells. The antibody staining was absent in TMAP siRNA-transfected cells and also abolished

\section{A}
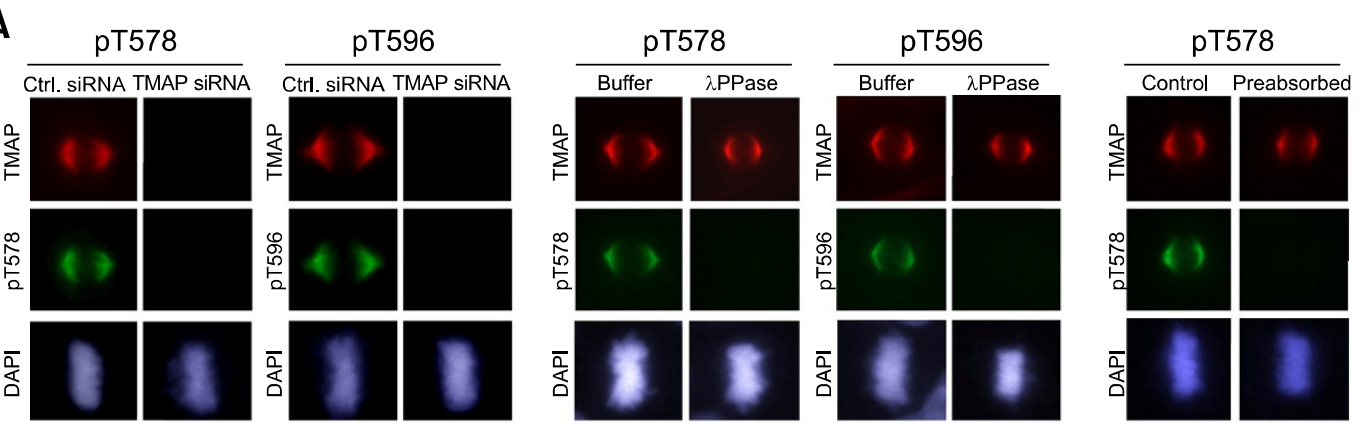

pT596

B Interphase Prophase

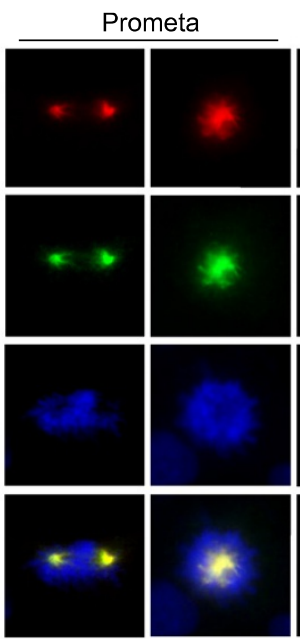

Metaphase Anaphase

Telophase Cytokinesis

TMAP
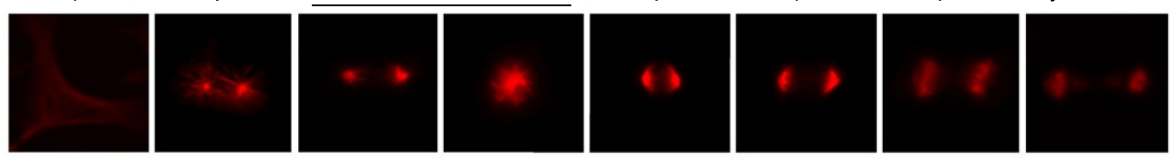

pT578
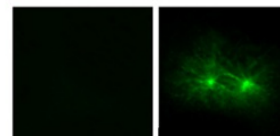

DAPI
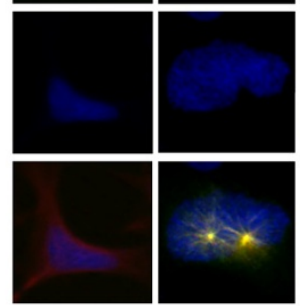

Interphase

Prophase

Prometa
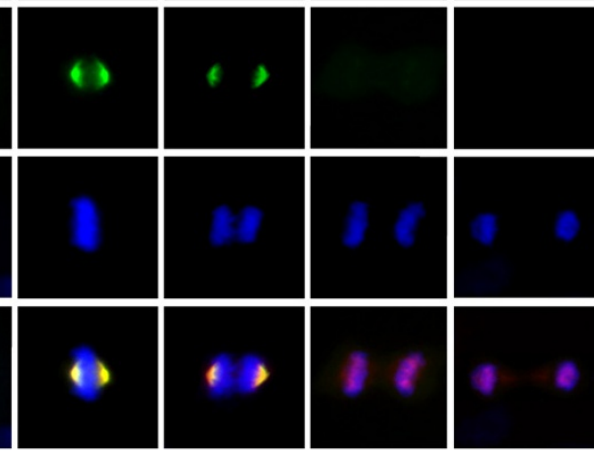

C
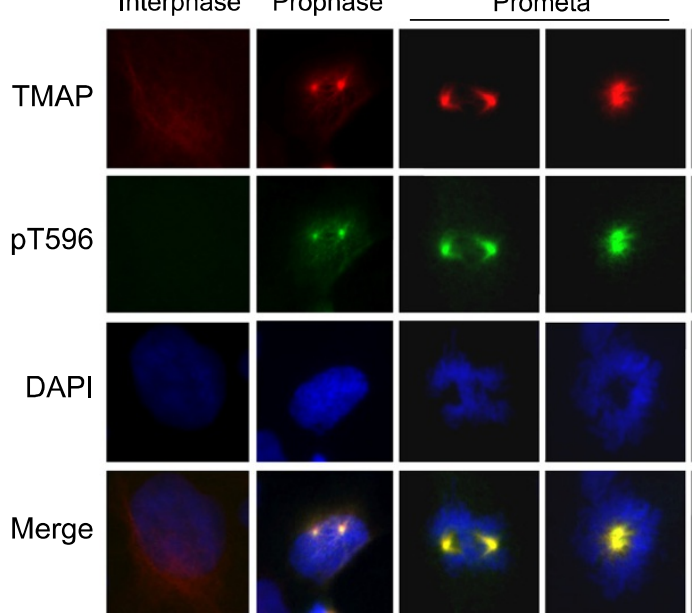

Metaphase Anaphase

Telophase Cytokinesis
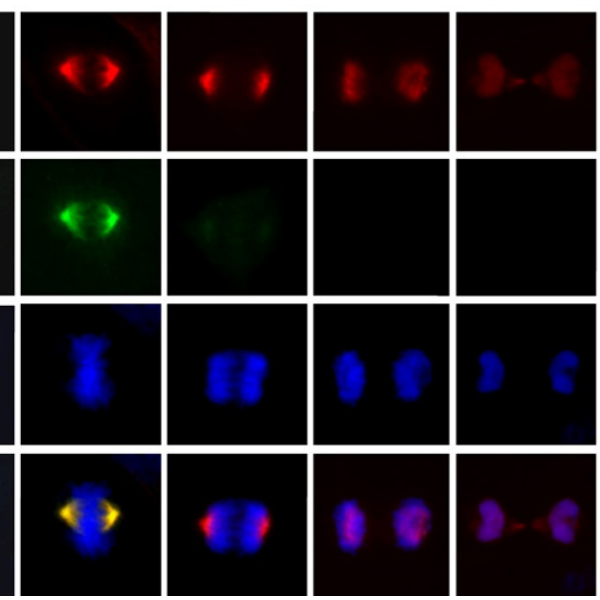

Figure 2. Immunofluorescence staining of phosphorylated forms of TMAP during mitosis. (A) Specificity of pT578 and pT596 antibody staining. HEK 293 cells were fixed and immunofluorescently stained for total TMAP (red) and pT578 or pT596 (green). DAPI staining (blue) shows DNA/chromosomes. Representative images of metaphase cells are shown here. siRNA-mediated depletion of endogenous TMAP (left panels), lambda phosphatase ( $\lambda$ PPase) treatment of fixed cells (center panels), and preabsorption of the antibody to its corresponding phospho-peptide (right panels) all abrogated pT578 or pT596 antibody staining of metaphase cells, showing the specificity of the antibody staining. (B) Phosphorylation of TMAP at Thr-578 during mitosis. HEK 293 cells were fixed and immunofluorescently stained for total TMAP (red) and pT578 (green). Representative images of interphase and mitotic cells at indicated stages are shown here. (C) Phosphorylation of TMAP at Thr-596 during mitosis. HEK 293 cells were immunofluorescently stained for total TMAP (red) and pT596 (green). 
by lambda phosphatase treatment after fixation, indicating that the antibodies specifically recognized phosphorylated forms of TMAP protein (Figure 2A). Moreover, preabsorption of each antibody with the corresponding phospho-peptide completely abolished the antibody staining, which further confirmed the phosphorylation site-specific binding of each antibody (Figure 2A).

Asynchronous HEK 293 cells were fixed and immunofluorescently stained with a mouse monoclonal antibody for TMAP and the polyclonal antibody for pT578 or pT596 in order to detect both total TMAP and the corresponding phosphorylated form of TMAP simultaneously (Figure 2B and 2C). During interphase, although TMAP-positive cells were frequently observed, no pT578 or pT596 staining was present. However, pT578 and pT596 staining began to appear in cells at early prophase in which chromosome condensation and separation of duplicated centrosomes were initiated. pT578 or pT596 staining remained relatively strong up to metaphase. After metaphase, pT578 staining intensity began to decrease and was still clearly visible in cells at anaphase, yet the staining was completely absent in cells at later stages of mitosis. In contrast, pT596 staining abruptly disappeared after metaphase and was no longer detected in cells at anaphase. During the window of pT578 or pT596 phosphorylation, the subcellular localizations of phosphorylated proteins were essentially identical to that of total TMAP protein. During prophase,
pT578 and pT596 forms of TMAP mainly localized to the separating centrosomes and adjacent microtubules, and during later stages, they were associated with the mitotic spindle apparatus, including spindle poles and spindle microtubules (Figure 2B and 2C).

\section{Effects of phosphorylation-deficient mutants of TMAP on mitotic progression}

Previously, we have shown that Cdk1/cyclin B1-mediated phosphorylation of TMAP at Thr-622 contributes to establishment of functional bipolar spindles. Introduction of T622A mutant form of TMAP, in which Thr-622 had been replaced with an alanine, significantly increases the frequency of cells with abnormal bipolar spindles, ultimately delaying the entry into anaphase (Hong et al., 2009b). In order to examine if Thr-578 and Thr-596 phosphorylation of TMAP during mitosis also functionally contributes to proper establishment of the spindle apparatus and thus normal progression of mitosis, we generated a phosphorylation-deficient mutant in which both Thr-578 and Thr-596 had been replaced with alanines (i.e., T578A/T596A). We then carried out time lapse video microscopic analyses of GFP-WT or GFP-T578A/T596A-expressing cells to observe any differences in the kinetics of mitotic progression. For comparison, GFP-T622A, which has previously shown to cause a significant delay in the metaphase to anaphase

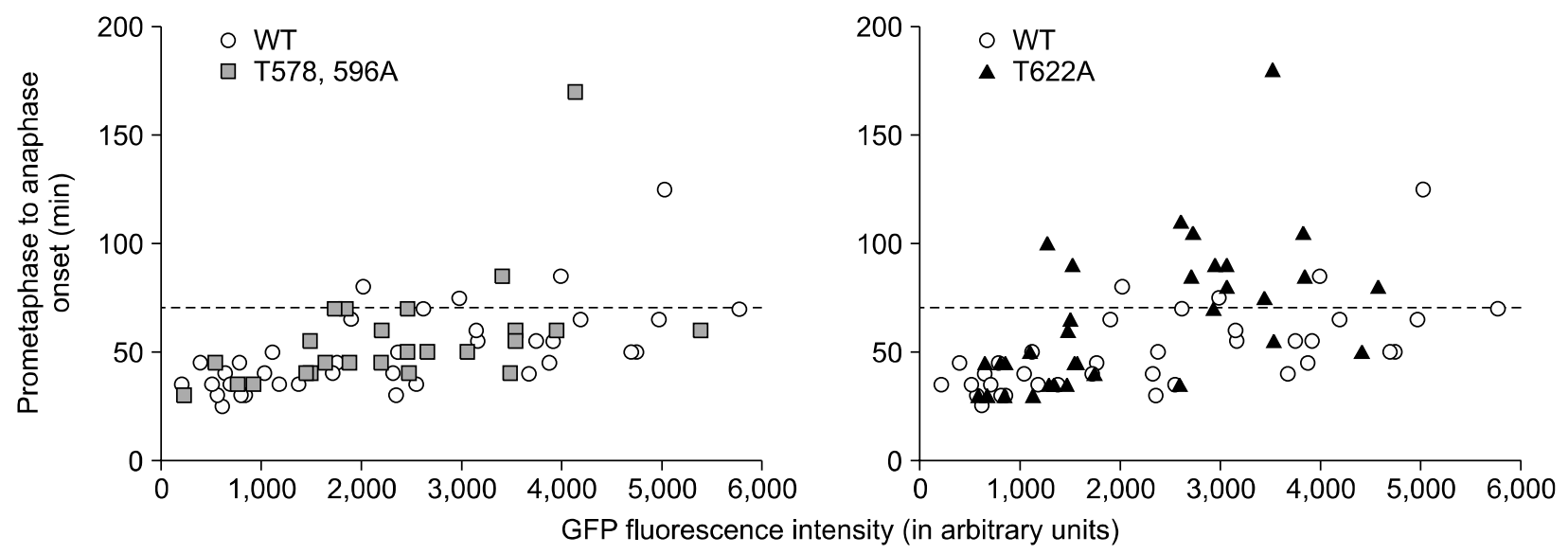

Figure 3. Effects of phosphorylation-deficient mutant forms of TMAP on progression of mitosis. HEK 293 cells introduced with GFP-WT or GFP-T578A/T596A or GFP-T622A TMAP were continuously observed at the same time using time lapse video microscopy. Analysis was limited to those that formed bipolar spindles and successfully completed mitosis without cytokinesis defects. A range of 24-36 cells were analyzed for each construct. The GFP fluorescence intensity was measured to compare the relative level of the GFP-fusion protein expression (x-axis), and the duration from prometaphase to anaphase entry was measured for each cell analyzed ( $y$-axis). The data set from a same experiment was divided and presented in two separate graphs to better compare the difference between T578A/T596A double mutant and T622A single mutant. As a result, WT data presented in two graphs are identical. The dotted line represents the average duration in GFP-T622A-expressing cells which was 73 min. Note that in contrast to the T622A mutant (filled triangles; right), the T578A/T596A double mutant form of TMAP (filled squares; left) did not induce significant delay in the entry into anaphase, compared to the WT counterpart (open circles). 
transition (Hong et al., 2009b), was used as a positive control. Since expression of WT or mutant TMAP at an excessive level both induced formation of monopolar spindles and prolonged mitotic arrest as previously reported (data not shown) (Hong et al., 2007), we focused our analysis on the cells which 1) expressed relatively low levels of the GFP-fusion proteins; 2) formed bipolar spindles; and 3) completed mitosis without cytokinesis defects. For the analysis, the GFP fluorescence intensity of the cells was measured to ensure that the cells were expressing similar levels of WT or mutant TMAP protein. In case of GFP-WT-expressing cells, the majority of cells expressing it at a relatively low level successfully formed bipolar spindles as they entered mitosis and took approximately $50.2 \pm 3.7 \mathrm{~min}$ on an average to enter anaphase (Figure 3 ). Consistent with the previous report, cells expressing GFP-T622A at a level similar to that of GFP-WT exhibited retardation of mitotic progression and took longer to enter anaphase $(72.9 \pm 9.7 \mathrm{~min}$ on an average), despite of the formation of bipolar spindles (Figure 3 ). However, in contrast to GFP-T622A, the average duration of pre-anaphase stages of mitosis in GFP-T578A/T596A-expressing cells (56.2 \pm 5.4 min) was not significantly different from that in GFP-WT control cells (Figure 3). These results suggest that unlike Thr-622 phosphorylation, phosphorylation at Thr-578 or Thr-596 may not make a significant contribution to proper establishment of bipolar spindles during mitosis.

\section{Discussion}

We have previously reported that TMAP is hyperphosphorylated near the C-terminus in mitotic cells and that at least four major sites (Thr-578, Thr-596, Thr-622, and Ser-627) are phosphorylated during mitosis (Hong et al., 2009b). Although the kinetics and mechanism of mitosis-specific phosphorylation of Thr-622 has been characterized, the precise kinetics of phosphorylation or the mechanisms of phosphorylation at remaining residues has not been previously examined in detail. Using phosphorylated residue-specific antibodies, the present study demonstrated that phosphorylation of TMAP at Thr-578 and Thr-596 indeed occurs specifically during mitosis. When synchronized HeLa cells were analyzed by Western blot, the window of Thr-578 and Thr-596 phosphorylation coincided with that of histone $\mathrm{H} 3$ phosphorylation (at serine 10) which is a well-known marker of mitosis (Hendzel et al., 1997). However, de-phosphorylation of Thr-578 and Thr-596 appeared to initiate slightly earlier than that of histone $\mathrm{H} 3$, suggesting that the window of Thr-578 and Thr-596 phosphorylation is restricted to relatively earlier phases of mitosis. This was also supported by the observation that the timing of de-phosphorylation was concomitant with cyclin B1 degradation which is initiated during metaphase to anaphase transition (Clute and Pines, 1999), suggesting that de-phosphorylation of Thr-578 and Thr-596 occurs following metaphase. This finding was finally confirmed by immunofluorescent staining of mitotic cells with phospho-specific antibodies. pT578 and pT596 staining was completely absent in interphase cells, yet became detectable starting at early prophase and sustained up to metaphase. After metaphase, the staining declined to an undetectable level, indicating that Thr-578 and Thr-596 become de-phosphorylated again as the cells progress into anaphase. The previously reported phosphorylation kinetics of Thr-622 is similar to those of Thr-578 and Thr-596 in that Thr-622 started to get phosphorylation at the onset of mitosis, remained high up to metaphase, and declined to an undetectable level some time after initiation of anaphase (Hong et al., 2009b). The phosphorylation kinetics of Thr-622 basically mirrors that of Thr-578 reported in the present study. However, in contrast to Thr-578 and Thr-622, Thr-596 appears to be relatively quickly de-phosphorylated at the onset of anaphase, and barely detectable or no pT596 staining was observed in anaphase cells. With respect to the kinetics of Thr-596 phosphorylation during mitosis, the present finding is consistent with our previous report (Hong et al., 2008). We speculate that phosphorylation events occurring at Thr-578 and Thr-596 may be under different mechanisms of regulation.

TMAP shows an interesting pattern of subcellular localization during mitosis. In interphase cells, TMAP localizes mainly to microtubules. During prophase, majority of protein is located at the separating centrosomes and adjacent microtubules, and during prometa/metaphase, TMAP is mainly found at the poles and microtubules of the spindle. Sometime after metaphase, however, it translocates to the chromosome region and is later found in newly formed nuclei and midbody microtubules (Hong et al., 2007). It is possible that cellular functions of TMAP are regulated by changes in its subcellular localization, and this process may be regulated by timely phosphorylation. For instance, there are reports that subcellular localizations of microtubule-associated motor proteins are regulated by Cdk1/cyclin B1-mediated phosphorylation during mitosis (Blangy et al., 1995; Ohsugi et al., 2003; Mishima et al., 2004). However, the subcellular 
localization of Thr-578- or Thr-596-phosphorylated TMAP was essentially identical to that of total TMAP. It is possible that phosphorylation status on Thr-578 or Thr-596 is not involved in regulation of subcellular localization of TMAP. This notion is consistent with our observation that the subcellular localizations of phosphorylation-deficient and phosphorylation-mimic mutant forms corresponding to Thr-578 or Thr-596 were not noticeably different from that of endogenous TMAP during interphase and mitosis (unpublished observations, H. J. Kim, K. U. Hong, C. D. Bae, and J. Park).

Functional importance of mitosis-specific phosphorylation of Thr-578 and Thr-596 remains unknown. We initially hypothesized that multiple phosphorylation occurring during mitosis may have similar effects on TMAP, changing its biochemical properties, for instance. Based on the previous observation that introduction of T622A mutant form of TMAP induces formation of structurally abnormal bipolar spindles (Hong et al., 2009b), we tested to see if mutations at Thr-578 and Thr-596 have similar effects on spindle structures and progression of mitosis. However, introduction of T578A/T596A double mutant form of TMAP did not significantly impair the progression of mitosis, suggesting that the assembly and functions of the bipolar spindle apparatus were not defective in these cells. This result suggests that phosphorylation of TMAP at Thr-579 or Thr-596 is not required or at least, does not make a significant contribution to the mitotic spindle-related functions of TMAP. Introduction of a triple TA mutant where Thr-578, Thr-596, and Thr-622 are all mutated did not result in further enhancement of the phenotype observed with T622A single mutant (i.e., abnormal bipolar spindle structures and delay in entry into anaphase) (unpublished observations, HJ Kim, KU Hong, CD Bae, and J Park). It appears that Thr-578/Thr-596 phosphorylation do not function in concert with Thr-622 phosphorylation during mitosis, at least under the experimental conditions tested in the present study. Of note, our preliminary study suggests that phosphorylation of Ser-627 is mediated by a different mitotic kinase and is involved in regulation of subcellular localization of TMAP during mitosis (unpublished observations, HJ Kim, KU Hong, CD Bae, and J Park). Based on these findings, multiple phosphorylation of TMAP during mitosis may not act in concert, but rather lead to diverse functional outcomes depending on the phosphorylation site.

The future studies will continue to explore the functional importance of Thr-578 and Thr-596 phosphorylation of TMAP in mitosis using phosphorylation-deficient and phosphorylation-mimic mutant forms of TMAP to further enhance our understanding of the mitosis-related roles of TMAP.

\section{Methods}

\section{Cell culture and transfection}

HeLa, HEK 293T, and HEK 293 cells were purchased from ATCC and cultured in DMEM containing 10\% FBS and antibiotics, and were subcultured every 3 days. For transfection of plasmid DNA, Lipofectamine Plus (Invitrogen) reagent was used according to the manufacturer's instructions. For transfection of siRNA, DharmaFECT1 (Dharmacon) was used according to the manufacturer's instructions.

\section{Expression constructs and siRNA}

Expression constructs for phosphorylation-deficient mutant forms of TMAP were generated using a QuikChange II Site-Directed Mutagenesis Kit (Stratagene) according to the manufacturer's instructions using pEGFP-C2 TMAP construct (Bae et al., 2003) as the template. Characterization of human TMAP-specific siRNA has been previously described (Jeon et al., 2006).

\section{Generation of pT578- or Thr-596-specific antibodies}

The following unphosphorylated and phosphorylated forms of synthetic peptides were produced at Peptron (Daejeon, Korea): CDPTHEVK(p)TPNTETRT-NH ${ }_{2}$ for Thr-578 and CIKYNVST(p)TPYLQSVK-NH ${ }_{2}$ for Thr-596. Rabbits were immunized with the KLH-conjugated phosphorylated peptide mixed with an adjuvant, TiterMax classic (TiterMax) four times at a 3-week interval by subcutaneous injections. Phosphorylation-specific antibodies were then affinity-purified from the serum in the following manner. Affinity columns were produced by conjugating unphosphorylated or phosphorylated peptide using SulfoLink Immobilization Kit (Pierce) according to the manufacturer's instructions. Firstly, crude serum was passed through the unphospho-peptide column to remove antibodies that recognize unphosphorylated epitope, and the resulting flow-through was passed through the phospho-peptide column to retain antibodies that are specific for the phosphorylated epitope. The eluent from the second column was tested for specificity and used for the present study.

\section{Preabsorption of phospho-antibodies}

Prior to immunoblotting or staining of the cells, $20 \mu \mathrm{l}$ of affinity-purified antibody was incubated with or without 10 $\mu \mathrm{g}$ of the corresponding phospho-peptide or none in $5 \%$ BSA in TBST for $1 \mathrm{~h}$ at room temperature. Control or preabsorbed antibody was then diluted further in $5 \%$ BSA in TBST for Western blot or immunofluorescent staining.

\section{Antibodies}

Generation and characterization of rabbit polyclonal antiserum to human TMAP has been previously described 
(Jeon et al., 2006). Mouse monoclonal antibody against human TMAP was produced as previously described (Hong et al., 2008). Mouse monoclonal antibodies against $\alpha$-tubulin (clone B-5-1-2) and cyclin B1 (clone GNS1) were purchased from Sigma and Santa Cruz, respectively. Rabbit polyclonal antibody against phospho-Histone $\mathrm{H} 3$ (Ser10) was purchased from Upstate. AlexaFluor 488-conjugated anti-rabbit IgG and Cy3-conjugated anti-mouse IgG were purchased from Molecular Probes and Rockland, respectively.

\section{Western blot and immunofluorescence staining}

These procedures were done as previously described (Hong et al., 2008).

\section{Lambda phosphatase treatment}

Asynchronous or nocodazole-arrested HeLa cells were lysed in RIPA buffer $(20 \mathrm{mM}$ Tris, $\mathrm{pH} 7.5,100 \mathrm{mM} \mathrm{NaCl}$, $0.5 \mathrm{mM}$ EDTA, 1\% NP-40, PMSF, aprotinin, and leupeptin) and centrifuged at $10,000 \times g$. The resulting lysate was untreated or treated with $\lambda$ phosphatase (New England Biolabs) in the presence of $2 \mathrm{mM} \mathrm{MnCl}_{2}$ for $1 \mathrm{~h}$ at $30^{\circ} \mathrm{C}$. The reaction was terminated by adding $5 \times$ Laemmli buffer. For immunofluorescent staining, formaldehyde-fixed cells were incubated with buffer alone (control) or with $\lambda$ phosphatase in the presence of $2 \mathrm{mM} \mathrm{MnCl} 2$ for $5 \mathrm{~h}$ at $30^{\circ} \mathrm{C}$.

\section{Double thymidine block and release}

HeLa cells were seeded at a density of $6-7 \times 10^{4}$ cells/35 $\mathrm{mm}$ dish, and on the following day treated with $2 \mathrm{mM}$ thymidine for $19-20 \mathrm{~h}$ for the first block. Cells were washed twice with PBS, released into fresh media for 8-10 h, and incubated with $2 \mathrm{mM}$ thymidine again for $16 \mathrm{~h}$. For the release, cells were washed twice in PBS, released into fresh media and harvested at indicated time points. Cells harvested at each time point were analyzed by both flow cytometry and Western blotting. For the flow cytometric analysis, cells were fixed in cold $70 \%$ ethanol overnight or longer. The fixed cells were spun down, washed in PBS containing $1 \% \mathrm{BSA}$, and incubated in the propidium iodide (PI) solution containing $50 \mu \mathrm{g} / \mathrm{ml} \mathrm{PI}, 0.1 \%$ sodium citrate, $0.3 \% \mathrm{NP}-40,50 \mu \mathrm{g} / \mathrm{ml}$ RNase A and $1 \times$ PBS for $1 \mathrm{~h}$ at $37^{\circ} \mathrm{C}$. DNA profiles of $\mathrm{PI}$-stained cells were obtained using a FACSCalibur system (BectonDickinson) and analyzed using CELLQuest software, ver. 3.3 (BectonDickinson).

\section{Time lapse video microscopy}

HEK 293 cells were transfected on a 6-well plate with a GFP-WT, GFP-T578A/T596A or GFP-T622A mutant TMAP expression plasmid. Twenty-four hours after transfection, 2-channel time lapse video microscopy was performed using a fully motorized Axiovert 200M microscope (Carl Zeiss), equipped with Axiocam HRm. Temperature and $\mathrm{CO}_{2}$ control was maintained using the Incubator S-M and Heating insert M06 controlled by Tempcontrol 37-2 and CTI-Controller 3700 . Both phase contrast and GFP fluore- scence images were acquired for $48 \mathrm{~h}$ with a lapse time of 5 min using AxioVision 4.3 software. Images were acquired using a $20 \times$ objective (LD Plan-Neofluar $20 \times 10.4$ Corr Ph2; Carl Zeiss). For quantification of GFP fluorescence intensity, the acquired images were analyzed using ImageJ $1.40 \mathrm{~g}$ (http://rsb.info.nih.gov/ij/). The GFP fluorescence intensity was measured from the area covering an entire mitotic cell and subtracted by the background fluorescence from a neighboring area.

\section{Acknowledgements}

This work was supported by a research grant from the National R\&D Program for Cancer Control, Ministry of Health and Welfare, Republic of Korea (grant 0720370).

\section{References}

Bae CD, Sung YS, Jeon SM, Suh Y, Yang HK, Kim YI, Park $\mathrm{KH}$, Choi J, Ahn G, Park J. Up-regulation of cytoskeletalassociated protein 2 in primary human gastric adenocarcinomas. J Cancer Res Clin Oncol 2003;129:621-30

Blangy A, Lane HA, d'Hérin P, Harper M, Kress M, Nigg EA. Phosphorylation by $\mathrm{p} 34 \mathrm{cdc} 2$ regulates spindle association of human Eg5, a kinesin-related motor essential for bipolar spindle formation in vivo. Cell 1995;83:1159-69

Clute P, Pines J. Temporal and spatial control of cyclin B1 destruction in metaphase. Nat Cell Biol 1999;1:82-7

Eichmuller S, Usener D, Dummer R, Stein A, Thiel D, Schadendorf D. Serological detection of cutaneous T-cell lymphoma-associated antigens. Proc Natl Acad Sci USA 2001;98:629-34

Hendzel MJ, Wei Y, Mancini MA, Van Hooser A, Ranalli T, Brinkley BR, Bazett-Jones DP, Allis CD. Mitosis-specific phosphorylation of histone $\mathrm{H} 3$ initiates primarily within pericentromeric heterochromatin during $\mathrm{G} 2$ and spreads in an ordered fashion coincident with mitotic chromosome condensation. Chromosoma 1997;106:348-60

Hong KU, Park YS, Seong YS, Kang D, Bae CD, Park J. Functional importance of the anaphase-promoting complexCdh1-mediated degradation of TMAP/CKAP2 in regulation of spindle function and cytokinesis. Mol Cell Biol 2007; 27:3667-81

Hong KU, Choi YB, Lee JH, Kim HJ, Kwon HR, Seong YS, Kim HT, Park J, Bae CD, Hong KM. Transient phosphorylation of tumor associated microtubule associated protein (TMAP)/cytoskeleton associated protein 2 (CKAP2) at Thr-596 during early phases of mitosis. Exp Mol Med 2008;40:377-86

Hong KU, Kim E, Bae CD, Park J. TMAP/CKAP2 is essential for proper chromosome segregation. Cell Cycle 2009a; 8:314-24

Hong KU, Kim HJ, Kim HS, Seong YS, Hong KM, Bae CD, Park J. Cdk1-cyclin B1-mediated phosphorylation of tumorassociated microtubule-associated protein/cytoskeleton associated protein 2 in mitosis. J Biol Chem 2009b;284: 
16501-12

Jeon SM, Choi B, Hong KU, Kim E, Seong YS, Bae CD, Park $\mathrm{J}$. A cytoskeleton-associated protein, TMAP/CKAP2, is involved in the proliferation of human foreskin fibroblasts. Biochem Biophys Res Commun 2006;348:222-8

Jin Y, Murakumo Y, Ueno K, Hashimoto M, Watanabe T, Shimoyama Y, Ichihara M, Takahashi M. Identification of a mouse cytoskeleton-associated protein, CKAP2, with microtubule-stabilizing properties. Cancer Sci 2004;95: 815-21

Maouche-Chretien L, Deleu N, Badoual C, Fraissignes P, Berger R, Gaulard P, Romeo PH, Leroy-Viard K. Identification of a novel cDNA, encoding a cytoskeletal associated protein, differentially expressed in diffuse large $B$ cell lymphomas. Oncogene 1998;17:1245-51

Mishima M, Pavicic V, Grüneberg U, Nigg EA, Glotzer M. Cell cycle regulation of central spindle assembly. Nature 2004; 430:908-13

Ohsugi M, Tokai-Nishizumi N, Shiroguchi K, Toyoshima YY, Inoue J, Yamamoto T. Cdc2-mediated phosphorylation of Kid controls its distribution to spindle and chromosomes. EMBO J 2003;22:2091-103

Whitfield ML, Sherlock G, Saldanha AJ, Murray JI, Ball CA Alexander KE, Matese JC, Perou CM, Hurt MM, Brown PO, Botstein D. Identification of genes periodically expressed in the human cell cycle and their expression in tumors. Mol Biol Cell 2002;13:1977-2000 